\title{
miRA: adaptable novel miRNA identification in plants using small RNA sequencing data
}

\author{
Maurits Evers ${ }^{1 *}$, Michael Huttner ${ }^{1}$, Anne Dueck ${ }^{2}$, Gunter Meister ${ }^{2}$ and Julia C. Engelmann ${ }^{1}$
}

\begin{abstract}
Background: MicroRNAs (miRNAs) are short regulatory RNAs derived from longer precursor RNAs. miRNA biogenesis has been studied in animals and plants, recently elucidating more complex aspects, such as non-conserved, species-specific, and heterogeneous miRNA precursor populations. Small RNA sequencing data can help in computationally identifying genomic loci of miRNA precursors. The challenge is to predict a valid miRNA precursor from inhomogeneous read coverage from a complex RNA library: while the mature miRNA typically produces many sequence reads, the remaining part of the precursor is covered very sparsely. As recent results suggest, alternative miRNA biogenesis pathways may lead to a more diverse miRNA precursor population than previously assumed. In plants, the latter manifests itself in e.g. complex secondary structures and expression from multiple loci within precursors. Current miRNA identification algorithms often depend on already existing gene annotation, and/or make use of specific miRNA precursor features such as precursor lengths, secondary structures etc. Consequently and in view of the emerging new understanding of a more complex miRNA biogenesis in plants, current tools may fail to characterise organism-specific and heterogeneous miRNA populations.

Results: miRA is a new tool to identify miRNA precursors in plants, allowing for heterogeneous and complex precursor populations. miRA requires small RNA sequencing data and a corresponding reference genome, and evaluates precursor secondary structures and precursor processing accuracy; key parameters can be adapted based on the specific organism under investigation. We show that miRA outperforms the currently best plant miRNA prediction tools both in sensitivity and specificity, for data involving Arabidopsis thaliana and the Volvocine algae Chlamydomonas reinhardtii; the latter organism has been shown to exhibit a heterogeneous and complex precursor population with little cross-species miRNA sequence conservation, and therefore constitutes an ideal model organism. Furthermore we identify novel miRNAs in the Chlamydomonas-related organism Volvox carteri.
\end{abstract}

Conclusions: We propose miRA, a new plant miRNA identification tool that is well adapted to complex precursor populations. miRA is particularly suited for organisms with no existing miRNA annotation, or without a known related organism with well characterized miRNAs. Moreover, miRA has proven its ability to identify species-specific miRNAs. miRA is flexible in its parameter settings, and produces user-friendly output files in various formats (pdf, csv, genome-browser-suitable annotation files, etc.). It is freely available at https://github.com/mhuttner/miRA.

Keywords: Sequencing data, miRNA identification, RNA secondary structure, Chlamydomonas reinhardtii, Small RNA sequencing, Next generation sequencing

\footnotetext{
*Correspondence: maurits.evers@anu.edu.au

1 Institute of Functional Genomics, University of Regensburg, Regensburg,

Germany

Full list of author information is available at the end of the article
} 


\section{Background}

MicroRNAs (miRNAs) are short endogenous non-coding RNA molecules that play an important role in regulating gene expression in many species within the animal and plant kingdoms. Since the discovery of miRNAs in Caenorhabditis elegans [1,2], detailed studies into transcription and the functional role of miRNAs across different species have lead to a complex picture of miRNA biogenesis and miRNA-associated regulatory pathways [3-5].

\section{A brief overview of miRNA biogenesis in plants}

The first step in miRNA biogenesis involves transcription of a primary miRNA transcript by RNA polymerase II. In canonical miRNA biogenesis, primary transcripts are then processed by the Dicer-like protein DCL1 to produce miRNA precursors (pre-miRNA) [6, 7]. Precursors exhibit double-stranded hairpin structures of varying length and levels of complexity (bulges, multiple shorter sub-hairpins etc.). While animal miRNA precursors are usually $80-100 \mathrm{nt}$ long and consist of simpler hairpin structures, plants and algae have a more heterogeneous precursor population, with pre-miRNAs of up to a few hundred nucleotides in length and often including additional shorter hairpins $[6,8]$. Following precursor formation, the pre-miRNA is exported to the cytoplasm, and processed by Dicer-like proteins to a $20-24$ nt long double-stranded RNA complex. Either the 5' or the 3' arm of the duplex may then be incorporated into the RNAinduced silencing complex (RISC), where it binds to a member of the AGO protein family $[9,10]$.

\section{Computational miRNA identification}

Computational miRNA identification based on nextgeneration sequencing (NGS) data involves identifying the genomic location of miRNA precursors, using small RNA expression primarily from the mature miRNA. Small RNA sequencing libraries typically also contain expression from other non-miRNA RNA species, such as other small RNAs with similar lengths and/or degradation products from protein-coding genes; computational miRNA identification requires filtering of these "background" signals in the sequencing data from sequencing reads associated with true miRNA expression. A common approach to do so is to use the fact that many miRNAs are evolutionarily conserved from species to species within the plant and the animal kingdoms. This gives rise to cross-species homologous miRNA families [11]. Database-supported computational tools for identifying novel miRNAs from sequencing data commonly apply a combination of (i) evaluating miRNA secondary structures, and (ii) ranking miRNA candidates by utilising existing annotation or evolutionary conservation of the mature miRNA sequence. Structure threshold parameters of most algorithms are often optimised based on animal miRNA precursor structures. Recent quantitative comparisons of the performance of various existing miRNA identification algorithms are given in [12] and [13].

Recent studies suggest that miRNA precursors often show more complex features and secondary structures, such as multiple mature/star duplexes per precursor, multiple hairpin loops, and tRNA precursor-like clover structures $[14,15]$. In plants, reports of mature miRNAs of different lengths (21 nt, $22 \mathrm{nt}$ and $24 \mathrm{nt}$ ) originating from longer (up to a few hundred nt) long precursors have shown that species-specific (non-conserved) miRNAs exist (see e.g. [16] and [17]), and play an important role in developing a better understanding of mechanisms related to miRNA origin and evolution $[6,18,19]$.

Here we introduce miRA for identifying miRNA precursors in plants and plant-like organisms (algae). miRA requires aligned small RNA sequencing data and a reference genome, and does not depend on existing miRNA annotation. Its main strength lies in the identification and charactersation of complex and non-homogeneous miRNA precursor populations. To our knowledge, miRA is also the first tool that allows to identify expression from multiple mature miRNA loci within one precursor. Within miRA, miRNA precursors are identified based on a set of species-specific constraints. Two key aspects of the algorithm presented in this paper are (1) not requiring crossspecies miRNA sequence conservation, and (2) allowing for a heterogeneous miRNA precursor population. This allows for a consistent characterisation of species-specific miRNAs and heterogeneous miRNA precursor structures in plants and algae, which in turn provides insight into the role of non-canonical miRNA biogenesis in these organisms.

We compare the performance of miRA with popular miRNA prediction tools using NGS data from two different organisms (Arabidopsis thaliana, Chlamydomonas reinhardtii), and identify novel miRNAs in the Chlamydomonas reinhardtii-related Volvocine algae Volvox carteri. The latter two organisms show a high degree of genome similarity, with recent results suggesting (1) very little conservation between miRNAs identified in both organisms, and (2) the existence of a heterogeneous miRNA precursor population [20, 21]. Both organisms therefore constitute an ideal example to apply and evaluate our algorithm. Results show an absence of miRNA conservation between both organisms, suggesting profoundly different, evolutionary-specific roles of miRNAs in Chlamydomonas reinhardtii and Volvox carteri.

\section{Implementation}

miRA uses high-throughput RNA sequencing data (typically small RNA sequencing data), and relies on a genomewide investigation of secondary hairpin structures. For 
reasons detailed in the introduction, we choose to be independent of cross-species sequence conservation. Therefore the process of identifying novel miRNAs depends (1) on the identification of a secondary structure that is consistent with that of a miRNA precursor, and (2) on a miRNA candidate precursor verification based on a precursor processing and read-coverage analysis.

Core modules of miRA are written in the $\mathrm{C}$ programming language, making full use of thread parallelisation on multi-core architectures using OpenMP [22]. miRA compiles and runs on any UNIX-based architecture (Linux, Mac OS X). To use miRA optimally, java, gnuplot and $\mathrm{HT}_{\mathrm{E}} \mathrm{X}$ should be installed. miRA is implemented using test-driven development, allowing every program function to be tested for its correct behaviour upon compilation of the source code, using the open-source MIT-licensed unit testing library 'testerino'. miRA includes customised versions of the RNAfold [23] and Varna [24] libraries, which were modified to allow the extraction of relevant data and remove unused code. ${ }^{1}$

Output files and plots are automatically generated, including (i) a GTF- and BED-formatted (see e.g. [25] for a description of the file formats) list of identified miRNA precursors and mature/star miRNAs for use in common genome browsers, (ii) a $\mathrm{AT}_{\mathrm{E}} \mathrm{X}$-based PDF report including secondary structure plots for every identified miRNA precursor, and (iii) a HTML-formatted table of all identified miRNA precursors including links to full miRNA reports that can be viewed in a web browser. The code can be downloaded from github https:/github.com/mhuttner/ miRA. Documentation and example files are included. The user typically runs miRA by specifying the speciesgroup under consideration (i.e. plants, algae). Alternatively the user may adjust key parameters individually. The modular structure of miRA enables the user to restart different sections of the pipeline. This allows for efficient computer time and resource management, in particular for jobs involving large genomes and/or sequencing data.

\section{Method}

In a three-stage process, we first identify genomic contigs based on small RNA sequencing data. In the second step, we analyse secondary structures for every cluster. Lastly, we verify that RNA sequencing data-based read coverage of miRNA precursor candidates is consistent with miRNA precursor processing resulting in the expression of one or more mature/star miRNA duplexes. We give details involving each step and a discussion of important key parameters (typeset in sans-serif) in the following sections ${ }^{2}$. Note that the time-consuming step of folding candidate sequences can be parallelised on multiple computer core architectures, by adjusting the parameter openmp_thread_count. If the OpenMP library is not present, this parameter will be ignored, and a single thread will be used for the sequence folding.

\section{Defining candidate clusters}

We require aligned strand-specific (small) RNA sequencing data in form of a sequence alignment/map (SAM) file, and a FASTA-formatted reference genome. In a first step towards identifying novel miRNA precursors, we generate a list of genomic regions based on and centred around a confined locus (contig) exceeding a threshold number of aligned and overlapping reads (cluster_min_reads). The latter was fixed at 10 reads for the analysis presented in this paper. This main expression contig is then extended at the $5^{\prime}$ and $3^{\prime}$ ends by an $F=200$ nt (default for cluster_flank_size) long flanking region, thus forming the candidate cluster as shown in Fig. 1 . The length of the 5'/3' end flank should be chosen such that candidate clusters are at least as long as miRNA precursors in the organism under investigation. The default value for cluster_flank_size should be suitable for most plant and plant-like organisms. Prior to extending contigs by the flanking regions, we merge neighbouring expression loci that lie less than 10 nucleotides (default for cluster gap size) apart, to form one combined contig. Finally we discard contigs exceeding a length of 2000 nt (default for cluster_max_length).

\section{Secondary structure investigation}

In the second step of the analysis, we investigate secondary structures that result from folding sequences of different lengths (i.e. different start and stop positions) located within the candidate cluster locus as defined in the previous analysis step, see Fig. 1. We require miRNA precursors to fulfil the following key criteria:

1. Existence and uniqueness of one optimal (i.e. minimal in its free energy) structure amongst all possible structures (c.f. [26]), the corresponding sequences of which have genomic start/stop coordinates located within the cluster's $5^{\prime} / 3^{\prime}$ flanking region.

2. A set of species-dependent secondary structure constraints, detailed in the following sections.

3. Statistical significance of the obtained optimal structure compared to structures resulting from random sequences with the same length and nucleotide distribution.

\section{Minimum in the secondary structure free energy surface}

Candidate cluster regions are folded using a modified version of RNALfold [23]. We calculate per-nucleotide (i.e. sequence length-normalised) minimum free energies MFE/nt for sequences with different start/stop coordinates within the $5^{\prime} / 3^{\prime}$ flanking region. The optimum sequence corresponds to the structure with the lowest $\mathrm{MFE} / \mathrm{nt}$. 


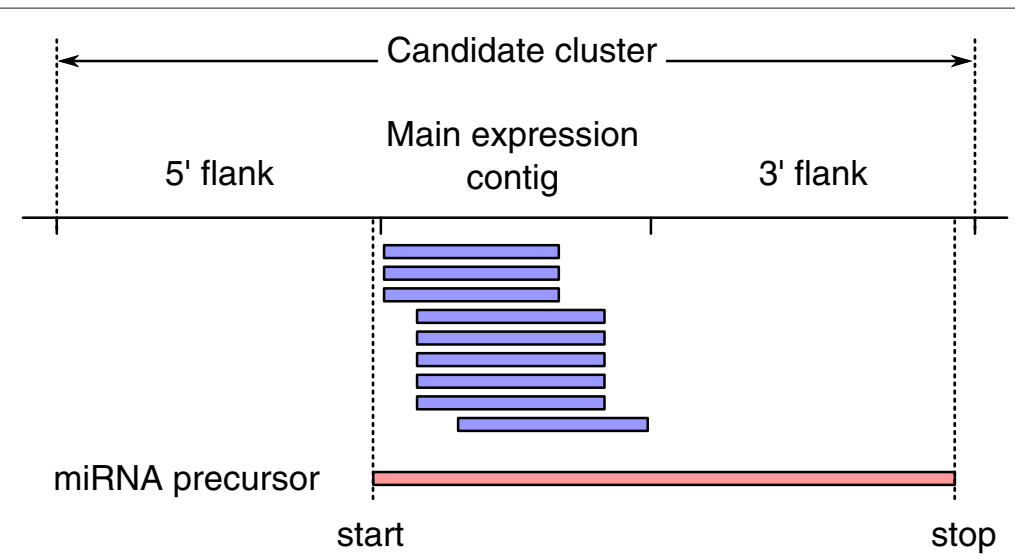

Fig. 1 Diagram illustrating the definition of a candidate cluster based on the main expression contig extended by a flanking region on the $5^{\prime}$ and $3^{\prime}$ end. Reads of the main expression contig are shown by the blue bars. The potential miRNA precursor with start/stop coordinates in the $5^{\prime} / 3^{\prime}$ flanking region is shown in red

\section{Secondary structure constraints}

We filter sequences corresponding to optimal secondary structures based on structure constraints that are consistent with characteristic features of miRNA precursors in the organism under investigation. Relevant key parameters are

1. the per-nucleotide minimum free energy of the secondary structure 'MFE/nt' (min_mfe_per_nt),

2. the number of terminal loops ' $N_{\text {term }}$ ' (max hairpin count), and

3. the length in nucleotides of the longest doublestranded segment allowing for two mismatches ' $L_{\mathrm{ds} \text {,max }}$ (min_double_strand_length) within the candidate structure.

It is important to note that these parameters are not necessarily independent of each other, as e.g. an increase in $L_{\mathrm{ds} \text {,max }}$ leads to a smaller minimum free energy MFE/nt of the resulting secondary structure.

We determined key parameters for different organisms based on an analysis of secondary structures of known miRNA precursors. To this extent, microRNA precursor sequences were obtained from miRBase [27], and their corresponding optimal secondary structures were analysed. We show the distribution of the minimum free energy per nucleotide of the miRNA precursor secondary structure (MFE/nt), length of the longest double-stranded segment within the precursor $\left(L_{\mathrm{ds}, \max }\right)$, and length of the precursor ( $L$ (precursor)) for Arabidopsis thaliana (Arabidopsis) and Chlamydomonas reinhardtii (Chlamydomonas) in Fig. 2. In comparison to miRNAs in Arabidopsis, Chlamydomonas miRNA precursors have on average longer double-stranded segments, smaller per nucleotide minimum free energies, and a precursor population with a larger variation in lengths.
The mean per-nucleotide minimum free energy decreases inversely with the sequence length $L$ as $\mathrm{MFE} / \mathrm{nt} \propto 1 / L$. This initially rapid decrease of MFE/nt with increasing sequence length may lead to precursor structures that include additional short maximally paired hairpins, in particular in organisms with longer precursors (plants, algae). While these sub-hairpins are not necessarily biologically realistic, the main structure without these extra hairpin(s) may still be consistent with that of a miRNA precursor. By choosing the number of terminal loop hairpins in the potential precursor structure to be $N_{\text {term }}<4$, we assure that such structures are not discarded prematurely.

\section{Statistical significance test}

In a next step, the statistical significance of sequences which pass all structure constraints is investigated. To obtain a statistical measure ( $p$-value) related to the significance of the secondary structure, we determine null distributions of the per-nucleotide minimum free energy $f(\mathrm{MFE} / \mathrm{nt})$ for every sequence that passes the structure constraints filter (max pvalue). This is done by randomly permuting nucleotides of the sequence using the Fisher-Yates algorithm (mono-nucleotide shuffling), and calculating corresponding minimum free energies. We account for sequence-specific nucleotide abundances by calculating null distributions for every sequence separately. The $p$-value related to the significance of the pernucleotide minimum free energy MFE/nt of the candidate sequence is then obtained from

$$
p=\int_{-\infty}^{\mathrm{MFE} / \mathrm{nt}} d\left(\mathrm{MFE} / \mathrm{nt}^{\prime}\right) f\left(\mathrm{MFE} / \mathrm{nt}^{\prime}\right) .
$$

For the calculation of the structure significance $p$-value no assumption is made upon the nature of the distribution; however it is interesting to note that the distribution 

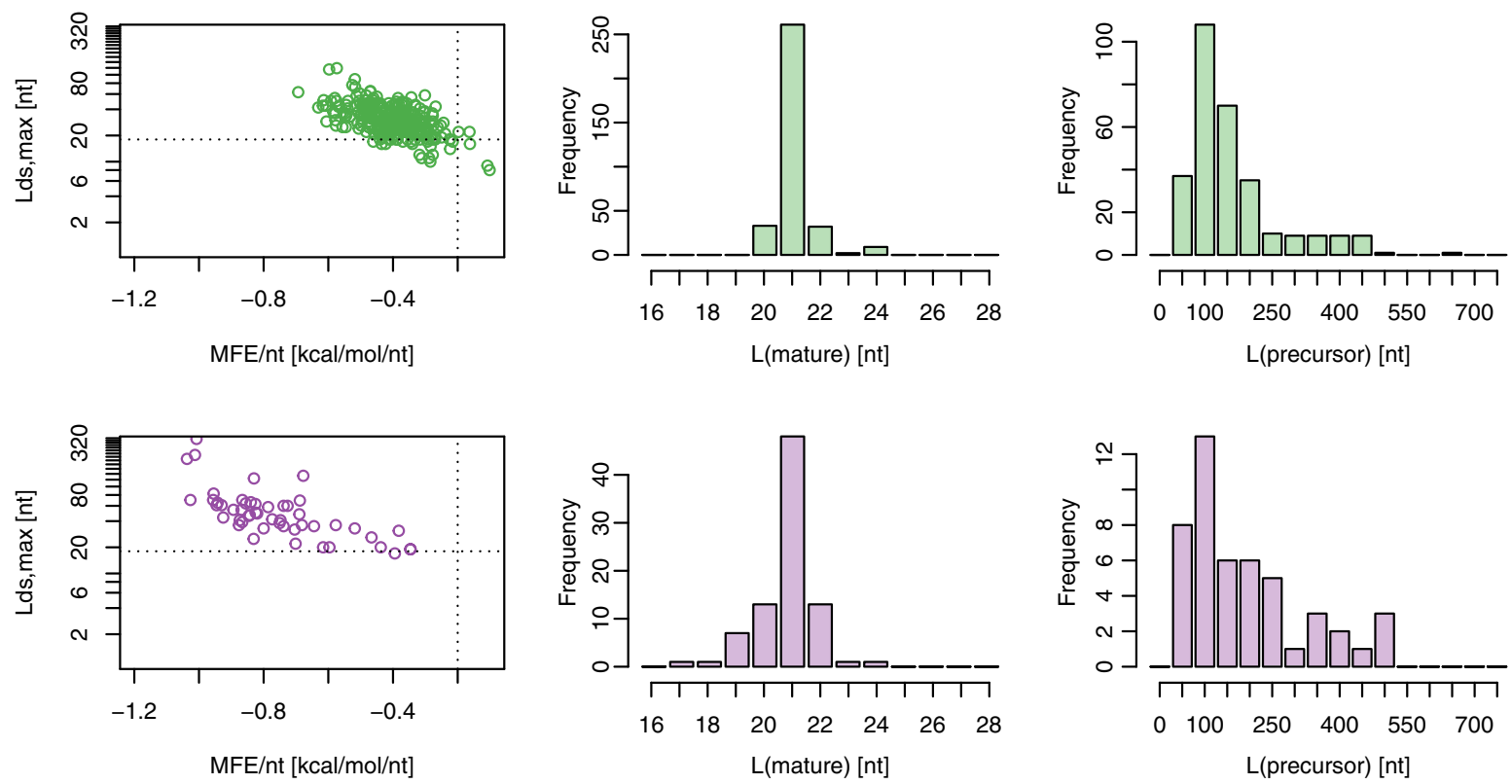

Fig. 2 Distributions of the minimum free energy per nucleotide of the miRNA precursor secondary structure (MFE/nt), length of longest double-stranded segment within the precursor $\left(L_{\mathrm{ds}, \max }\right)$ and length of the precursor ( $L$ (precursor)) for known miRNA precursors in Arabidopsis thaliana (top three panels) and Chlamydomonas reinhardtii (bottom three panels). All precursor sequences were obtained from miRBase ([27]). Secondary structure calculations were performed using RNAfold ([23]). The dotted lines mark the thresholds adopted for the identification of miRNAs in the corresponding organisms

of MFE/nt for random sequences is fairly well approximated by a normal distribution. Di-nucleotide shuffling does not lead to different results. This can be attributed to the fact that mono-nucleotide and di-nucleotide shuffling of longer sequences ( $\gtrsim 100 \mathrm{nt}$ ) lead to the same distribution of MFE/nt. Results of the structure significance analysis for two candidate sequences are summarised in Figure $\mathrm{S} 1$ of the Additional file 1.

\section{miRNA precursor verification}

In the last step of the analysis pipeline, we use a read coverage-based verification procedure to investigate whether observed expression from the miRNA precursor locus is consistent with that of a miRNA precursor containing at least one mature miRNA. The verification process consists of a series of adjustable constraints on the identified mature/star miRNA sequences, which are related to precursor processing accuracy. For each miRNA precursor candidate, we validate that

1. a mature miRNA locus can be defined with the following properties:

(a) Sharp edges in strand-specific read coverage at the $5^{\prime}$ or $3^{\prime}$ end, containing $>$ min_coverage of the full miRNA precursor coverage.

(b) Length min_duplex_length $\leq$ $L$ (mature) $<\max$ duplex length, and
2. taking into account DCL processing leading to $2 \mathrm{nt} 3$ ' overhangs, a complementary star miRNA segment of length min_duplex_length $\leq L$ (star) $<$ max_duplex_length exists.

Additionally, we may require that (adopted from [8]):

3. the fraction of paired nucleotides within the mature miRNA locus is $\geq$ min_paired_fraction,

4. the mature miRNA segment does not fold back on itself, and

5. the mature miRNA segment has $<4$ adjacent unpaired nucleotides at nucleotide positions 3 ...L (mature) -3 (allow_three_mismatches), and $<3$ adjacent unpaired nucleotides at the $5^{\prime}$ and $3^{\prime}$ end of the duplex (allow_two_terminal_mismatches).

We give a list of adopted key parameters for different organisms in Table S1 of the Additional file 1. Parameters are consistent with values discussed in various other publications such as $[8,28]$.

The underlying alignment data used in the verification process can be different from the alignment data used for the initial identification of main expression contigs. This allows for an independent cross-verification of candidate miRNAs. 


\section{Results and discussion}

We perform a benchmark analysis of miRA and up-todate, plant-suitable sequence data-based miRNA prediction tools. We use the prediction tools miRDP (formerly called miRDeep-P) [29] and miR-PREFeR [28], the latter of which was demonstrated to show superior performance compared to other existing tools [28]. Attempts to use miRExpress [30] and miReNA [31] were unsuccessful: miRExpress failed to compile on recent Linux and Mac OS X builds, and miReNA failed to identify any miRNA from the NGS data; while these tools have demonstrated their applicability in regards to miRNA identification, results highlight the need for a flexible and easy-to-use miRNA identification method such as miRA.

We use both simulated and experimental data to evaluate and compare the performance of miRA and miRPREFeR. For each method and data set we determine the recall rate (i.e. sensitivity or true positive rate) $\mathrm{RR}=\mathrm{TP} /(\mathrm{TP}+\mathrm{FN})$, where $\mathrm{TP}$ and $\mathrm{FN}$ are the number of true positives and false negatives, respectively. Additionally, the analysis of results based on the simulated data allows us to investigate the specificity (i.e. true negative rate) $\mathrm{SPC}=\mathrm{TN} /(\mathrm{FP}+\mathrm{TN})$, where TN and FP are the number of true negatives and false positives, respectively. Details and results involving the different data sets are given in the following sections.

\section{Simulated data}

We simulate miRNA and background expression from protein-coding genes (the latter constituting false positives) of the Volvocine algae Chlamydomonas reinhardtii (Chlamydomonas) using Flux Simulator [33]. For 20 known miRNA precursors with unambiguous strandassignment from [8], we generate reads for the mature and star loci. Expression strengths of mature/star loci within the precursors, as well as expression strengths of the precursors themselves are sampled uniformly. For the latter we choose a minimum expression strength to make sure that all miRNAs are expressed. The set of Flux Simulator parameters is given in Table S2 of the Additional file 1. Expression of protein-coding genes is generated using the catalogue of 14,595 annotated protein-coding genes for the Chlamydomonas reference genome version 3.0 from [34]. Simulated reads were then mapped to the Chlamydomonas reference genome [34] using tophat/bowtie2 $[32,35]$. The reference genome version matches the version used for annotating miRNAs in [8]. It is important to emphasize that the simulated data set corresponds to a challenging scenario, constituting a high background of degraded transcripts from protein-coding genes and only a small number of miRNAs.

It is interesting to note that annotated Chlamydomonas miRNAs constitute a heterogeneous precursor population (see [8]), with 50 of all precursors having more than two $21 \mathrm{nt}$ long main expression loci. Furthermore, folding

Table 1 Comparison of recall rates (RR) of different NGS-based miRNA identification tools using various data sets

\begin{tabular}{|c|c|c|c|c|c|c|c|}
\hline \multirow{2}{*}{$\begin{array}{l}\text { Organism and } \\
\text { library reference }\end{array}$} & \multirow{2}{*}{$\begin{array}{l}\text { Identification } \\
\text { method }\end{array}$} & \multicolumn{2}{|c|}{ miRNA reference data } & \multirow[t]{2}{*}{$N_{\text {recall }}$} & \multirow[t]{2}{*}{ RR } & \multirow[t]{2}{*}{$N_{\text {tot }}$} & \multirow[t]{2}{*}{ SPC } \\
\hline & & Source & $N_{\text {ref }}$ & & & & \\
\hline \multicolumn{8}{|c|}{ Chlamydomonas reinhardtii } \\
\hline Simulated & miRA & Molnar et al. [8] & 20 & 12 & 0.60 & 19 & 1.0 \\
\hline Simulated & miR-PREFeR & Molnar et al. [8] & 20 & 0 & 0.00 & 0 & 1.0 \\
\hline Loizeau et al. [36] & miRA & Molnar et al. [8] & 47 & 39 & 0.83 & 281 & - \\
\hline Loizeau et al. [36] & miRDP & Molnar et al. [8] & 47 & 14 & 0.30 & 964 & - \\
\hline Loizeau et al. [36] & miR-PREFeR & Molnar et al. [8] & 47 & 29 & 0.62 & 60 & - \\
\hline Molnar et al. [8] & miRA & Molnar et al. [8] & 15 & 12 & 0.80 & 175 & - \\
\hline Molnar et al. [8] & miRDP & Molnar et al. [8] & 15 & 7 & 0.47 & 51 & - \\
\hline Molnar et al. [8] & miR-PREFeR & Molnar et al. [8] & 15 & 3 & 0.20 & 6 & - \\
\hline \multicolumn{8}{|l|}{ Arabidopsis thaliana } \\
\hline Pooled Athl-2 [28] & miRA & miRBase & 246 & 122 & 0.50 & 517 & - \\
\hline Pooled Athl-2 [28] & miRDP & miRBase & 246 & 80 & 0.12 & 695 & - \\
\hline Pooled Athl-2 [28] & miR-PREFeR & miRBase & 246 & 119 & 0.48 & 138 & - \\
\hline \multicolumn{8}{|l|}{ Volvox carteri } \\
\hline Novel data & miRA & - & 0 & - & - & 213 & - \\
\hline
\end{tabular}

We compare the performance of miRA, miRDP [29], and miR-PREFeR [28] using simulated and experimental algae NGS data (Chlamydomonas reinhardtii and Volvox carteri), and Arabidopsis thaliana NGS data. Details of the simulated data are given in the text. We determine the number of reference miRNAs for each library by requiring a minimum expression of 10 reads for each known reference miRNA. The source and number $N_{\text {ref }}$ of known miRNAs for the different organisms are given in columns 3 and 4 . $N_{\text {recall }}$ gives the number of identified known miRNAs. $N_{\text {tot }}$ gives the total number of identified miRNAs. For the simulated data we provide the specificity (SPC) in the last column 
of most annotated precursor sequences yields minimum energy-associated secondary structures with complex features such as e.g. additional shorter hairpin loops, additional bulges etc. Therefore simulated Chlamydomonas data provides an excellent test data set to investigate the performance of miRNA identification algorithms given a complex miRNA population.

We determine recall rates and specificities of miRA, and compare results with those of miR-PREFeR. Results are summarised in Table 1. miR-PREFeR fails to identify any of the miRNAs.

\section{Experimental data}

\section{Chlamydomonas reinhardtii}

We use two different Chlamydomonas small RNA sequencing libraries from [36] and [8]. Corresponding adapter-trimmed and quality-filtered sequencing data were obtained from the gene expression omnibus (GEO), accession numbers GSE32457 (http://www.ncbi.nlm.nih. gov/geo/query/acc.cgi?acc=GSE32457) and GSE7575 (http://www.ncbi.nlm.nih.gov/geo/query/acc.cgi?acc=GS E7575), and converted to the FASTA format. Resulting reads were again mapped to the Chlamydomonas reference genome version 3.0 using tophat/bowtie2. The reference genome version was chosen such that results allowed for a direct comparison of derived miRNA loci with those from [8].

We identify Chlamydomonas miRNA precursors using miRA, and determine recall rates based on the list of known miRNAs from [8]. We use Molnar's list of annotated miRNAs instead of Chlamydomonas data from miRBase due to the existence of duplicate entries in the miRBase data, primarily from [20]. Molnar et al. list 31 Chlamydomonas miRNAs with unambiguous precursor strands, and 19 miRNAs with ambiguous precursor strands. We exclude miRNA precursors from the lists that are located on unassembled bonus scaffolds since we do not include these extra scaffolds in our Chlamydomonas reference genome. The final number of reference miRNAs used for calculating recall rates is determined by requiring a minimum expression of 10 reads per known reference miRNA, and the resulting numbers are given in Table 1.

Results based on miRA, miRDP and miR-PREFeR for both data sets are summarised in Table 1 . miRA recall rates for both data sets are comparable and $\geq 80 \%$. The larger number of novel miRNAs derived from the data in [36] compared to those from [8] is related to the larger sequencing depth of the former. This difference in sequencing depth is also reflected in the different numbers of expressed reference miRNAs. Recall rates for $\mathrm{mRDP}$ and miR-PREFeR are significantly smaller, dropping well below $50 \%$ in some cases; in a direct comparison of miRDP and miR-PREFeR, the former seems to perform better with low sequencing depth data, while

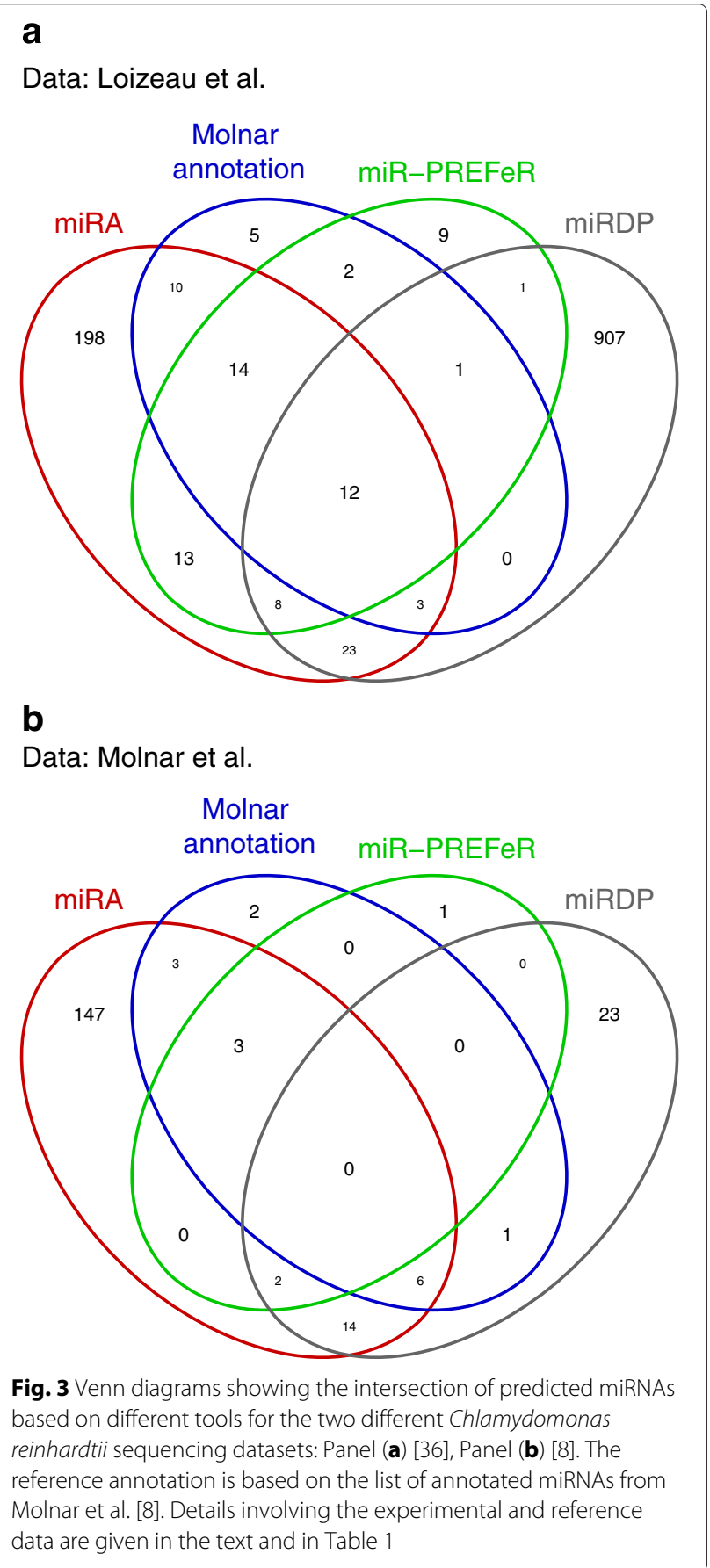

miR-PREFeR outperforms miRDP with deeper sequencing data. A detailed comparison of identified miRNAs using miRA, miRDP and miR-PREFeR is given in Fig. 3.

We are able to identify many novel Chlamydomonas miRNA precursors, many of which show expression profiles consistent with the generation of two and more mature miRNAs from the same precursor. Corresponding precursor structures range from up to $500 \mathrm{nt}$ long 
single hairpin structures, and up to $700 \mathrm{nt}$ long multiple hairpin structures. Examples of complex miRNA precursors are given in Figure S2 of the Additional file 1. Structural features of novel miRNA precursor structures often include multiple bulges and larger terminal loops; these complex structures in connection with multiple mature miRNA expression and overall longer precursors are believed to be responsible for miRPREFeR and miRDP not being able to successfully identify the corresponding expressed loci with potential miRNA precursors.

A complete list of identified Chlamydomonas miRNA precursors is provided in Table S3 of the Additional file 1. Distributions of the key parameters per-nucleotide minimum free energy of the miRNA precursor secondary structure (MFE/nt), length of the longest double-stranded segment in the precursor $\left(L_{\mathrm{ds}, \max }\right)$, length of the primary (i.e. most strongly expressed) mature miRNA ( $L$ (mature)), and length of the miRNA precursor ( $L$ (precursor)) for the identified (known and novel) Chlamydomonas miRNA precursors based on the data from [36] are summarised in the three middle panels of Fig. 4. They show good agreement with corresponding distributions derived from miRBase Chlamydomonas miRNA precursors as shown in the bottom panel of Fig. 2. We see from Fig. 4 that precursor lengths vary significantly, extending to up to $\sim 700 \mathrm{nt}$. Corresponding secondary structures confirm the existence of a complex and heterogeneous miRNA precursor population.

\section{Arabidopsis thaliana}

We use an Arabidopsis thaliana (Arabidopsis) library containing two samples that was used in the miR-PREFeR publication [28]. Details of the Athl-2 datasets can be found in the supplements of [28]. Pooled reads were mapped to the TAIR10 reference genome [37] using

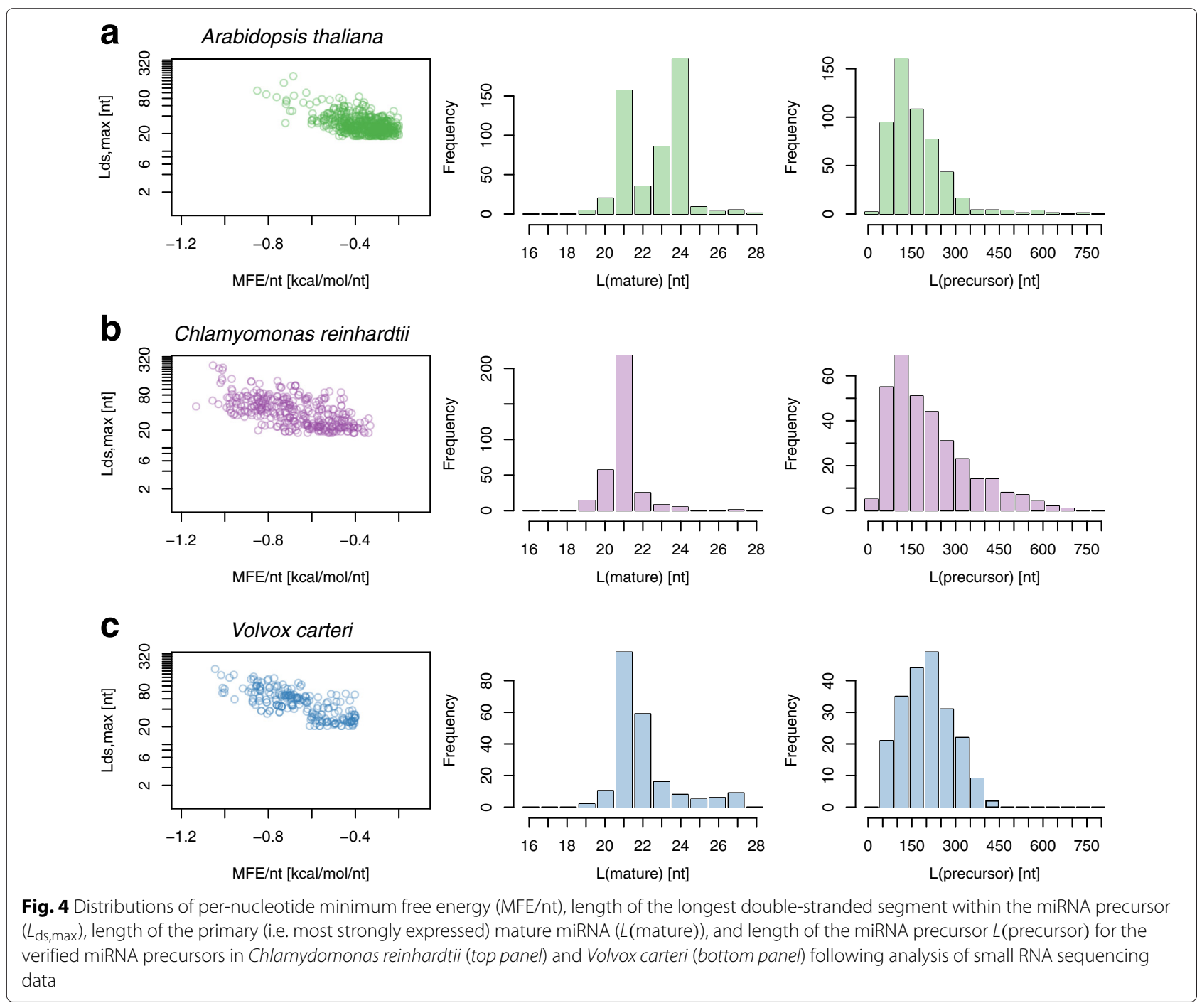


tophat/bowtie2. A list of known reference miRNAs was downloaded from miRBase and filtered by requiring a minimum expression of 10 reads per miRNA.

We compare the performance of miRA, miRDP and miR-PREFeR in Table 1. Recall rates of miRA and miRPREFeR are near identical, with miRA predicting more novel miRNAs. This is believed to be related to miRPREFeR's requirement of the existence of star-sequence associated reads, whereas miRA does not impose a minimum expression threshold on the star sequence. We show the distribution of key parameters MFE/nt, $L_{\mathrm{ds} \text {, max }}$, $L$ (mature) and $L$ (precursor) in the top three panels of Fig. 4. The performance of miRDP is significantly lower than that of miRA and miR-PREFeR, confirming results from [28].

It is interesting to note that in comparison to results for Chlamydomonas, the Arabidopsis precursor population is more homogeneous, showing a narrower length distribution and fewer complex secondary structures. The length distribution of mature miRNAs in Arabidopsis shows two characteristic peaks at $21 \mathrm{nt}$ and $24 \mathrm{nt}$. Only a small fraction $(\sim 15 \%)$ of the loci corresponding to the $24 \mathrm{nt}$ long sequences are repeat element-associated, which may support the association of these sequences with siRNAs/ta-siRNAs. This suggests that the majority of identified mature miRNAs of different lengths should be attributed to alternative miRNA biogensis pathways such as miRNA precursor processing by different members of the Dicer-like enzymes (see e.g. $[16,38]$ ). The corresponding Volvocine distributions show the existence of a single peak at $21 \mathrm{nt}$. This suggests a change in or the absence of complex Dicer-like processing in green algae.

\section{Volvox carteri}

We use miRA to identify novel miRNAs in the Volvocine organism Volvox carteri (Volvox). We use small RNA sequencing data derived from Volvox somatic cells during their vegetative cycle (GEO accession number GSE58703). Reads were mapped to the Volvox reference genome version 9.0 ([39]) using tophat/bowtie2. Distributions of key parameters equivalent to those discussed in the previous sections for Chlamydomonas and Arabidopsis are shown in the bottom panel of Fig. 4. A complete list of identified novel Volvox miRNAs is given in Table S4 of the Additional file 1.

To validate the identication of novel miRNAs in Volvox, Northern blots were performed on three randomly picked miRNAs from the list of identified novel miRNAs. Expression was confirmed for all three miRNA candidates, and the resulting blots are shown in Figure S3 of the Additional file 1.

The identified Volvox miRNAs show large similarities in the distribution of per-nucleotide free energy, and precursor and mature miRNA lengths compared to Chlamydomonas results. The slightly bi-modal distribution of MFE/nt suggests the existence of a plant-like and an algae-like miRNA sub-population, further confirming the existence of a heterogeneous population of miRNAs as was already the case for Chlamydomonas. Identified mature miRNA sequences show no similarity to identified mature miRNA sequences in Chlamydomonas. Given the large degree of similarity between the two genomes, the absence of any miRNA-conservation between the two closely related organisms is surprising, see also [21].

\section{Conclusion}

miRA presents a new conservation-independent miRNA identification algorithm, which identifies genomic locations of miRNA precursors based on (small RNA) sequencing data of plants and plant-like organisms (algae). miRA is particularly suited to investigate heterogeneous miRNA precursor populations. Identification of miRNA precursors occurs through an evaluation of corresponding secondary structures and subsequent precursor processing accuracy. Our method has three key features: First, it allows for the identification of miRNAs in species with little or no miRNA conservation. Second, it enables a consistent investigation of both species-specific and homologous miRNAs in different organisms. Third, it allows for the identification of miRNA precursors with complex and heterogeneous secondary structures, such as precursors including e.g. additional sub-hairpins or multiple mature/star miRNA duplexes.

\section{Availability and requirements}

Project name: miRA

Project home page: https://github.com/mhuttner/miRA Operating system(s): Any Unix-based system (MacOS, Linux)

Programming language: $\mathrm{C}$

Other requirements: Optional requirements: Java 1.6+, LaTeX, gnuplot

License: GNU GPL

\section{Endnotes}

${ }^{1}$ RNAfold is invoked from the main program, with parameters being passed directly to RNAfold as part of miRA's main routine.

${ }^{2}$ Values given in the following sections correspond to default values, and may be changed by the user.

\section{Additional file}

Additional file 1: miRA default parameters. (PDF 2333 kb)

Competing interests

The authors declare that they have no competing interests. 


\section{Authors' contributions}

ME implemented a first draft of miRA, performed analyses and wrote the manuscript. $\mathrm{MH}$ implemented the public version of miRA. AD interpreted miRA results and helped with parameter selection and calibration. GM provided Volvox sequencing data. ME and JCE conceived of the tool and the study. JCE contributed to manuscript writing. All authors read and approved the final manuscript.

\section{Acknowledgments}

The authors acknowledge funding from the Deutsche

Forschungsgemeinschaft (SFB 960), the Bavarian Genome Research Network (BayGene), and the Bavarian Biosystems Network (BioSysNet)

\section{Author details}

${ }^{1}$ Institute of Functional Genomics, University of Regensburg, Regensburg, Germany. ${ }^{2}$ Biochemistry Center Regensburg (BZR), Laboratory for RNA Biology, University of Regensburg, Regensburg, Germany.

\section{Received: 26 June 2015 Accepted: 22 October 2015}

\section{Published online: 05 November 2015}

\section{References}

1. Lee RC, Feinbaum RL, Ambros V. The C. elegans heterochronic gene lin-4 encodes small RNAs with antisense complementarity to lin-14. Cell. 1993;75:843-54

2. Reinhart BJ, Slack FJ, Basson M, Pasquinelli AE, Bettinger JC, Rougvie AE, et al. The 21-nucleotide let-7 RNA regulates developmental timing in Caenorhabditis elegans. Nature. 2000;403(6772):901-6.

3. Bartel B, Bartel DP. MicroRNAs: at the root of plant development? Plant Physiol. 2003;132:709-17.

4. Bartel DP. MicroRNAs: genomics, biogenesis, mechanism, and function. Cell. 2004;116:281-97.

5. Ameres SL, Zamore PD. Diversifying microRNA sequence and function. Nat Rev Mol Cell Biol. 2013;14:475-88.

6. Jones-Rhoades MW, Bartel DP, Bartel B. MicroRNAS and their regulatory roles in plants. Annu Rev Plant Biol. 2006;57:19-53.

7. Winter J, Jung S, Keller S, Gregory RI, Diederichs S. Many roads to maturity: microRNA biogenesis pathways and their regulation. Nat Cell Biol. 2009;11(3):228-34.

8. Molnár A, Schwach F, Studholme DJ, Thuenemann EC, Baulcombe DC miRNAs control gene expression in the single-cell alga Chlamydomonas reinhardtii. Nature. 2007:447(7148):1126-1129.

9. Meister $\mathrm{G}$. Argonaute proteins: functional insights and emerging roles. Nat Rev Genet. 2013;14:447-59.

10. Dueck A, Meister G. Assembly and function of small RNA - Argonaute protein complexes. Biol Chem. 2014;395:611-29.

11. Weber MJ. New human and mouse microRNA genes found by homology search. FEBS J. 2005;272(1):59-73.

12. Li Y, Zhang Z, Liu F, Vongsangnak W, Jing Q, Shen B. Performance comparison and evaluation of software tools for microRNA deep-sequencing data analysis. Nucleic Acids Res. 2012;40(10):4298-305.

13. Williamson V, Kim A, Xie B, McMichael GO, Gao Y, Vladimirov V. Detecting miRNAs in deep-sequencing data: a software performance comparison and evaluation. Briefings in Bioinforma. 2013;14(1):36-45.

14. Babiarz JE, Ruby JG, Wang Y, Bartel DP, Blelloch R. Mouse ES cells express endogenous shRNAs, siRNAs, and other Microprocessor-independent, Dicer-dependent small RNAs. Genes Dev. 2008;22:2773-785.

15. Röther S, Meister G. Small RNAs derived from longer non-coding RNAs. Biochimie. 2011;93(11):1905-1915.

16. Schwab $R$, Voinnet $O$. RNA silencing amplification in plants: size matters Proc Natl Acad Sci USA. 2010;107(34):14945-14946.

17. Liu YX, Wang M, Wang XJ. Endogenous small RNA clusters in plants Genomics, proteomics \& bioinforma. 2014;12(2):64-71.

18. Berezikov E. Evolution of microRNA diversity and regulation in animals. Nat Rev Genet. 2011;12:846-60.

19. Shi B, Gao W, Wang J. Sequence fingerprints of microRNA conservation. PLOS ONE. 2012;7(10):48256.

20. Zhao T, Li G, Mi S, Li S, Hannon GJ, Wang XJ, et al. A complex system of small RNAs in the unicellular green alga Chlamydomonas reinhardtii. Genes Dev. 2007;21(10):1190-1203.
21. Li J, Wu Y, Qi Y. MicroRNAs in a multicellular green alga Volvox carteri. Sci China Life Sci. 2014:57(1):36-45.

22. The OpenMP Architecture Review Board. The OpenMP Application Program Interface. http://openmp.org/wp/ Accessed date June 2015.

23. Hofacker IL, Fontana W, Stadler PF, Bonhoeffer LS, Tacker M, Schuster P. Fast Folding and Comparison of RNA Secondary Structures. Monatsh Chem. 1994;125:167-88

24. Darty K, Denise A, Ponty Y. VARNA: Interactive drawing and editing of the RNA secondary structure. Bioinforma. 2009;25(15):1974-1975.

25. UCSC Genome Bioinformatics. UCSC Genome Bioinformatics. http://genome.ucsc.edu/FAQ/FAQformat Accessed date June 2015.

26. Bonnet $E$, Wuyts J, Rouz e $P$, Van de Peer $Y$. Evidence that microRNA precursors, unlike other non-coding RNAs, have lower folding free energies than random sequences. Bioinforma. 2004;20(17):2911-917.

27. Kozomara A, Griffiths-Jones S. miRBase: annotating high confidence microRNAs using deep sequencing data. Nucleic Acids Res. 2014;42: 68-73.

28. Lei J, Sun Y. miR-PREFeR: an accurate, fast and easy-to-use plant miRNA prediction tool using small RNA-Seq data. Bioinformatics. 2014:30: 2837-2839.

29. Yang $X$, Li L. miRDeep-P: a computational tool for analyzing the microRNA transcriptome in plants. Bioinforma. 2011;27(18):2614-615.

30. Wang WC, Lin FM, Chang WC, Lin KY, Huang HD, Lin NS. miRExpress: analyzing high-throughput sequencing data for profiling microRNA expression. BMC Bioinforma. 2009.

31. Mathelier A, Carbone A. MIReNA: finding microRNAs with high accuracy and no learning at genome scale and from deep sequencing data. Bioinforma. 2010:26:2226-234

32. Langmead B, Salzberg SL. Fast gapped-read alignment with Bowtie 2 Nature Methods. 2012;9(4):357-9.

33. Griebel T, Zacher B, Ribeca P, Raineri E, Lacroix V, Guigo R, et al. Modelling and simulating generic RNA-Seq experiments with the flux simulator. Nucleic Acids Res. 2012;40(20):10073-10083.

34. Merchant SS, Prochnik SE, Vallon O, Harris EH, Karpowicz SJ, Witman $\mathrm{GB}$, et al. The Chlamydomonas genome reveals the evolution of key animal and plant functions. Science. 2007;318(5848):245-50

35. Kim D, Pertea G, Trapnell C, Pimentel H, Kelley R, Salzberg SL. TopHat2: accurate alignment of transcriptomes in the presence of insertions, deletions and gene fusions. Genome Biology. 2013;14(4):36

36. Loizeau K, Qu Y, Depp S, Fiechter V, Ruwe H, Lefebvre-Legendre L, et al. Small RNAs reveal two target sites of the RNA-maturation factor Mbb1 in the chloroplast of Chlamydomonas. Nucleic Acids Res. 2014;42:3286-297.

37. Lamesch P, Berardini TZ, Li D, Swarbreck D, Wilks C, Sasidharan R, et al. The Arabidopsis Information Resource (TAIR): improved gene annotation and new tools. Nucleic Acids Res. 2012;40(Database issue):1202-10.

38. Vazquez F, Blevins T, Ailhas J, Boller T, Meins F. Evolution of Arabidopsis MIR genes generates novel microRNA classes. Nucleic Acids Res. 2008:36(20):6429-438.

39. Prochnik SE, Umen J, Nedelcu AM, Hallmann A, Miller SM, Nishii I, et al. Genomic Analysis of Organismal Complexity in the Multicellular Green Alga Volvox carteri. Science. 2010;329(5988):223-6.

\section{Submit your next manuscript to BioMed Central and take full advantage of:}

- Convenient online submission

- Thorough peer review

- No space constraints or color figure charges

- Immediate publication on acceptance

- Inclusion in PubMed, CAS, Scopus and Google Scholar

- Research which is freely available for redistribution

Submit your manuscript at www.biomedcentral.com/submit
C Biomed Central 